(D) Check for updates

Cite this: Polym. Chem., 2020, 11 275

Received 22nd July 2019, Accepted 5th September 2019

DOI: $10.1039 / c 9 p y 01089 k$

rsc.li/polymers

\section{Investigating the self-assembly and shape transformation of poly(ethylene glycol)-b-poly (D,L-lactide) (PEG-PDLLA) polymersomes by tailoring solvent-polymer interactions $\uparrow$}

\author{
Imke A. B. Pijpers, ${ }^{a}$ Fenghua Meng, ${ }^{b}$ Jan C. M. van Hest*a and \\ Loai K. E. A. Abdelmohsen (D)*a
}

The assembly of amphiphilic block copolymers in well-defined polymer nanoparticles is an intricate interplay between polymer composition, method of assembly and the properties of the organic solvent used to dissolve the polymer building blocks. Remarkably, the role of organic solvent composition has been much less studied in comparison to the other two parameters. Here we have systematically investigated the effect of organic solvent composition, namely, the ratio between tetrahydrofuran and dioxane, on the self-assembly of PEG-PDLLA copolymers into polymer vesicles and their subsequent shape change in different topologies. Uncovering such physical basis unlocks new opportunities for the development of complex nanoparticles without the need of extended polymer engineering processes.

\section{Introduction}

The molecular engineering of polymeric architectures from amphiphilic copolymers has been documented since the findings of Eisenberg. ${ }^{1}$ Despite the large body of research currently done on the subject, achieving control over the morphology of such supramolecular structures has proven to remain challenging. ${ }^{2-6}$ Although we have much more detailed understanding of the morphological features on the nanoscale, it is observed that apparently small changes in chemical composition (such as polymer chain length, polydispersity, composition, blending etc.) and fabrication methodology have significant consequences for the resulting particle morphology. ${ }^{7-14}$ External factors, such as temperature, $\mathrm{pH}$ and osmotic pressure contribute to the dynamicity of the system and,

\footnotetext{
${ }^{a}$ Department of Bio-Organic Chemistry, Institute of Complex Molecular Systems (ICMS), Eindhoven University of Technology, Het Kranenveld 14, 5600 MB Eindhoven, The Netherlands. E-mail: j.c.m.v.hest@tue.nl, l.k.e.a.abdemohsen@tue.nl

${ }^{b}$ Biomedical Polymers Laboratory, College of Chemistry, Chemical Engineering and Materials Science, Soochow University, Suzhou 215123, PR China

$\dagger$ Electronic supplementary information (ESI) available. See DOI: 10.1039/ c9py01089k
}

indeed, to the final supramolecular structure. ${ }^{15-20}$ With this in mind, researchers from diverse backgrounds have sought to unravel the physicochemical basis for copolymer self-assembly in order to enhance our fundamental understanding of these systems. Often, however, only the relationship between the polymeric composition and the resulting assembly is considered. Remarkably, the (organic) solvent that is crucial in the formation and subsequent manipulation (such as resizing/ shape change) of the assemblies is an underexposed element in the entire structure formation. Commonly, the choice of solvent is based on its miscibility with water, and its ability to molecularly dissolve the copolymeric material. In this paper, we show that the composition of the organic solvent ensures the creation of a multitude of vesicular topologies (both during formation and manipulation), whereby small variations can have major consequences. A better understanding of this effect increases the reproducibility of the formation process and gives access to a wider range of structures.

The self-assembly of polymersomes can be performed in a wide variety of manners, one of which is the solvent switch methodology, which involves dissolving a copolymer into a 'good' solvent followed by slow addition of a 'poor' solvent. ${ }^{16,21}$ Increasing the amount of the poor solvent in the mixture leads to hydrophobic membrane-formation - as a result of copolymer reorganization. The outcome of such solvent switch methodology largely depends on the respective properties of the organic solvent and its interaction with the polymer building blocks. In general, good solvents promote material-solvent interactions, wherein polymers can be molecularly dissolved. On another note, non-solvents, or poor solvents, stimulate polymer-polymer (hydrophobic) interactions where polymer chains are folded into each other and become selfknotted. $^{22,23}$ Indeed, the solvation and de-solvation of polymers and the way they influence polymer aggregation play a significant role in driving the self-assembly process toward the formation of bilayered membrane or micellar structures, for instance. ${ }^{24}$ Moreover, solvents impact the way the polymers are packed in the self-assembled structures, which in turn affects 


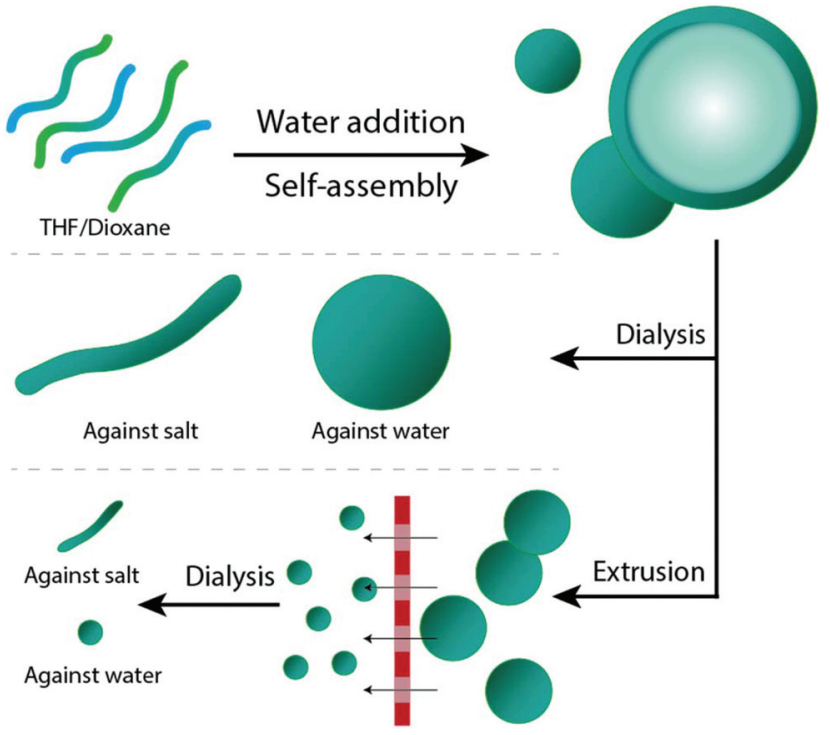

Fig. 1 Schematic describing the self-assembly of PEG-PDLLA polymers into polymersomes and subsequent morphology control. First, polymers are assembled into polymersomes. Then, particles are either dialyzed against water to yield spherical particles or salt to induce shape transformation into tubes; or particles are first extruded and then dialyzed to form spherical polymersomes or tubes.

the way in which they undergo subsequent shape change process. $^{25-28}$

In our group, we have experience with the self-assembly and the shape transformation of various copolymers, one of which is poly(ethylene glycol)- $b$-poly(D,L-lactide) (PEG-PDLLA). By applying the above mentioned solvent switch methodology, such copolymers assemble into spherical polymersomes (Fig. 1). Upon their exposure to hypertonic conditions, these spherical structures can undergo an osmotic-induced shape transformation process into prolates or oblates, depending on the chemical composition of the copolymer used during the initial assembly. ${ }^{29}$ Resizing such polymersomes was shown to be feasible by repeated extrusion - a process aiming to yield smaller sized nanoparticles. ${ }^{30}$

Although the changes in the chemical composition of PEG-PDLLA affect the self-assembly and shape transformation, it is evident that the choice of solvent is crucial to bring reproducibility to this system. Herein, we provide an insight to our initial hypothesis regarding the influence of the solvent composition on the self-assembly of PEG-PDLLA polymersomes and subsequent shape transformation process - a study that aims to enhance our fundamental understanding in order to bring control and reproducibility to these systems, broadening our accessibility to new structures.

\section{Results and discussion}

A mixture of tetrahydrofuran (THF) and dioxane (in different ratios) is typically used as solvent for PEG-PDLLA copolymers, and as a plasticizer for the resulting bilayered membrane. ${ }^{19,29}$
In order to study how the composition of the organic solvent affects the behaviour of $\mathrm{PEG}_{22}-\mathrm{PDLLA}_{45}$ during the self-assembly and shape transformation processes, we systematically varied the ratio of THF and dioxane during the initial polymersome formation. Thereafter, the results of both the self-assembly process and shape transformation were investigated.

First, $\mathrm{PEG}_{22}-\mathrm{PDLLA}_{45}$ with $D \leq 1.2$ copolymer was synthesized following a previously published method. ${ }^{19}$ In short, ring-opening polymerization (ROP) was carried out with $\mathrm{D}, \mathrm{L}^{-}$ lactide, using methoxy PEG-OH as macroinitiator and 1,8-diazabicyclo(5.4.0)undec-7-ene (DBU) as organocatalyst. The resulting chain length and $D$ were determined by ${ }^{1} \mathrm{H}-\mathrm{NMR}$ and Gel Permeation Chromatography (GPC), respectively (Fig. S1-3†). Previously, such copolymers were shown to form spherical polymersomes, and upon their exposure to hypertonic conditions, elongated tubes could be formed. Initial polymersome formation was performed by dissolving the polymers in a mixture of THF and dioxane with a ratio of $4: 1(\mathrm{v} / \mathrm{v})$, followed by slow addition of an equal volume of water to the mixture. Subsequently, the organic solvent was removed via dialysis against MilliQ at $4{ }^{\circ} \mathrm{C}$. Inverting the ratio of THF : dioxane to $1: 4$ during the self-assembly process resulted in unreproducible polymersome formation and major formation of aggregates - even after removing organic solvents, which was expected to force membrane rearrangement (Fig. 2).

This result indicates that indeed THF is crucial to the formation of stable PEG-PDLLA polymersomes, as the increased polarity of THF increases repulsive (electrostatic) interactions between polymer chains, which is necessary for the formation of ordered polymeric membranes with well-defined organization. As such, undefined polymer aggregates were formed by
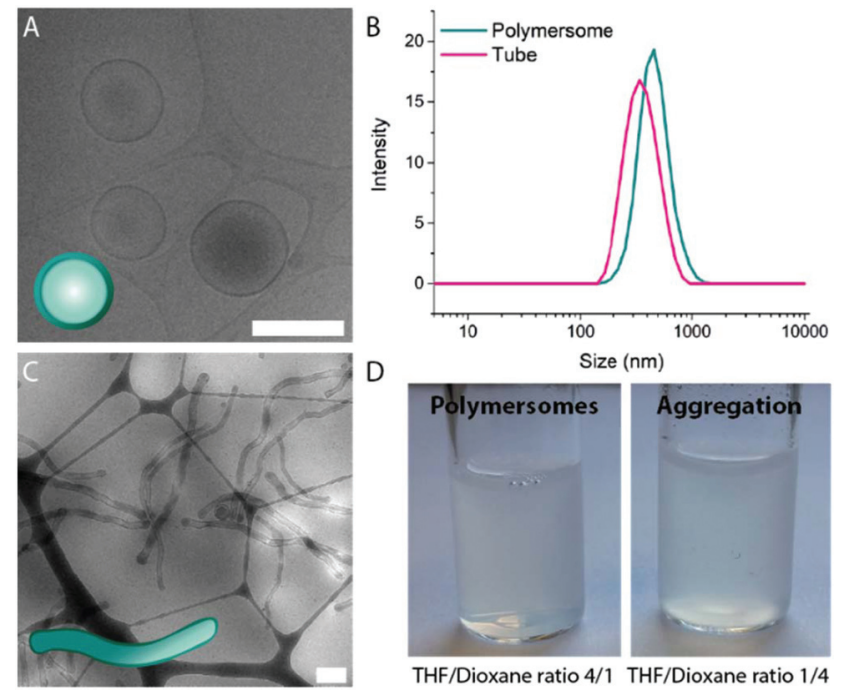

Fig. 2 Cryo-TEM images of spherical polymersomes (A) and tubular polymersomes (C). (B) Hydrodynamic size of polymersomes and tubes measured by DLS. Please note the difference in size in polymersomes and tubes which is a result of the volume reduction following the shape transformation process. (D) Formation of polymersomes (left) and aggregation (right) with varying THF: dioxane ratio. Scale bars are $500 \mathrm{~nm}$. 
Table 1 Summary of experimental data from varying the THF : dioxane solvent ratio during polymersome formation and shape transformation

Solvent composition scheme

\begin{tabular}{|c|c|c|c|c|c|}
\hline & \multicolumn{2}{|c|}{ Ratio solvents } & \multirow[b]{2}{*}{ Initial morphology } & \multirow[b]{2}{*}{ Structure after shape transformation } & \multirow[b]{2}{*}{ Mean aspect ratio } \\
\hline & Dioxane & Tetrahydrofuran & & & \\
\hline 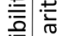 & 2 & 8 & Spheres & Tubes & 21.60 \\
\hline$\frac{\pi}{2}$ & 3 & 7 & Spheres & Tubes & 21.22 \\
\hline 要 & 5 & 5 & Spheres & Tubes & 33.49 \\
\hline ·䨔 & 7 & 3 & Aggregation & Aggregation & \\
\hline
\end{tabular}

increasing polymer-polymer charge interactions - a result of decreasing the amount of THF during the self-assembly in the initial polymer solution.

Systematic variation of the THF and dioxane ratios in the initial polymer solution, and accordingly, in the final assembled-polymersome solution resulted in clear differences in the formation of spherical vesicles. High amounts of THF resulted in the self-assembly of spherical polymersomes, while addition of increasing volumes of dioxane induced aggregation in the resulted structures (Table 1). Contrarily, the presence and quantity of dioxane in the polymer mixture proved to influence the shape transformation of the spherical particles (Fig. 3 and S4B, $5 \dagger$ ). At the first instance, it was clear that the size of the formed spherical polymersomes was bigger at higher dioxane ratios (Fig. S4†). Moreover, the length of the resulting tubes increases at a higher dioxane to THF ratio. Additionally, aggregates were observed when no THF was present in the system, confirming the importance of THF in modulating the stability of the assembled membrane. From cryo-TEM image analysis, it is apparent that the mean aspect ratio of the tubular structures increased with increasing dioxane content (Fig. 3F and S6†).

Interestingly, the presence of nested vesicles, closed stomatocytes, became increasingly apparent at higher THF ratios (Fig. S5†). Experimental details are given in Table 1.

Indeed, both solvents play their respective part in the selfassembly process; their individual properties, such as dielectric constant and solubility parameter, have significant consequences for how PEG and PDLLA chains are folded in the membrane. The dielectric constant $(\varepsilon)$ can be used as an indication of solvents' polarity, with values for THF and dioxane being $\varepsilon=7.6$ and $\varepsilon=2.3$, respectively. The higher polarity of THF results in increased repulsive interactions between the hydrophilic chains of the membrane. Dioxane, having a lower polarity, leads to weaker repulsive interactions. ${ }^{31}$ As a consequence, the ratio between both THF/dioxane in the solvent mixture determines the polymer conformation (or stretching), and therefore membrane architecture. ${ }^{22}$ In general, solventinduced repulsive (electrostatic) interactions among polymeric chains of copolymeric assemblies originate predominantly from electrostatic repulsion between hydrophilic polymer
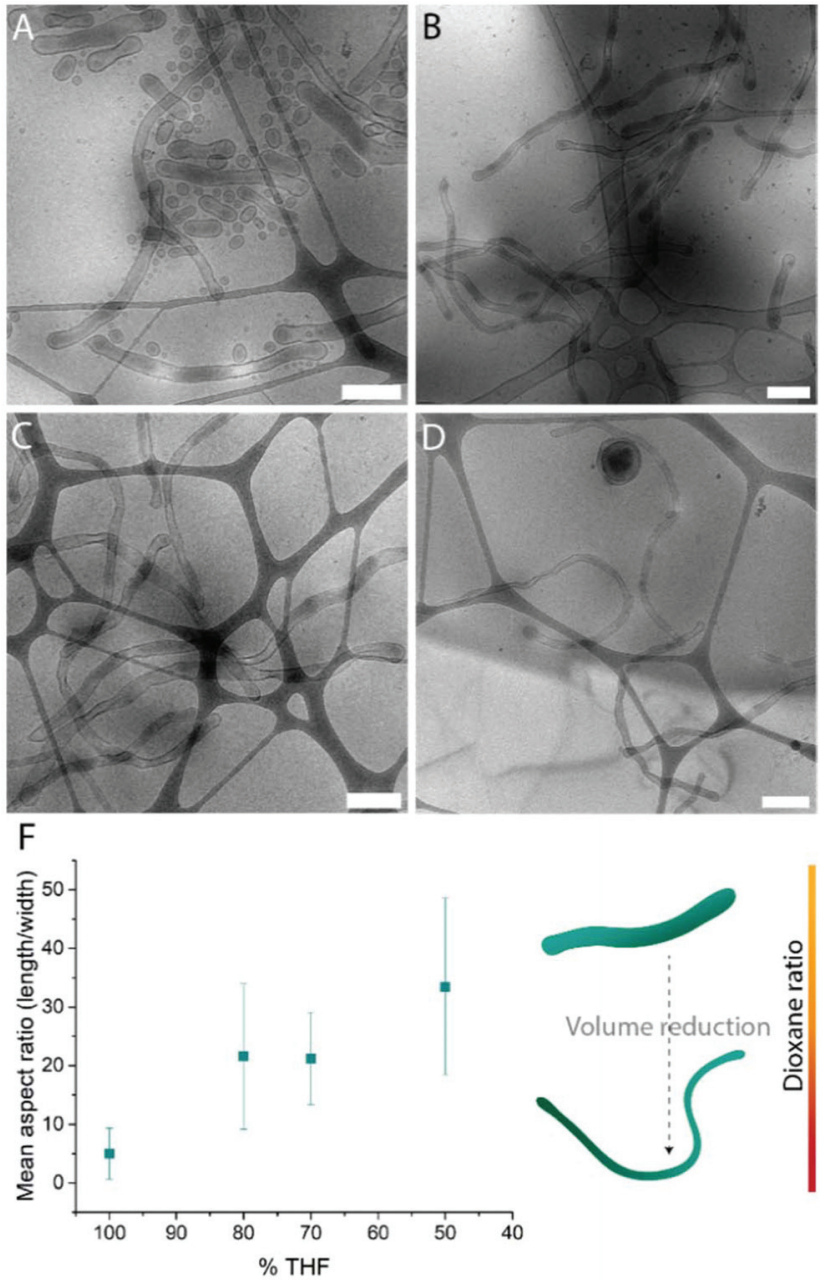

Fig. 3 Cryo-TEM images of tubes formed with THF: dioxane ratio (v/v\%) (A) $100: 0$, (B) $80: 20$, (C) $70: 30$ and (D) $50: 50$. Mean aspect ratio measured from image analysis (F). Scale bars are $500 \mathrm{~nm}$.

chains, ${ }^{31}$ and possibly from adsorption of negative ions onto the hydrophobic chains. ${ }^{32}$ The effect of various solvents with distinct dielectric constants on the resulting vesicle morphology was established by Yu and Eisenberg, where varying 
amounts of DMF, THF and dioxane yielded vesicles, spheres or rods with various sizes. ${ }^{33}$ These results were explained to be a consequence of a combination of effects that involve interactions between the solvent used and the copolymers, which in turn affects both polymer folding in solution and the repulsion of chains in the membrane of the resulting assembly. Moreover, the organic solvent composition influences the dimensions of the polymer during self-assembly (i.e. packing parameter) and the critical water content necessary to initiate self-assembly. Our hypothesis states that based on the $\varepsilon$ values, an increase in THF content should lead to the selfassembly of stable structures. On the other hand, dioxane can modulate the rigidity of the polymer chains in the membrane, allowing subsequent shape transformation process to occur. From our research into vesicular shape changes of polymersomes, we understand that both membrane flexibility and vesicle volume reduction are deciding factors in whether a spherical polymersome undergoes shape transformation. Originally, the shape change of $\mathrm{PEG}_{22}-\mathrm{PDLLA}_{45}$ spherical polymersomes was induced by dialyzing flexible spherical polymersomes (in $50 \%$ organic solvent $\mathrm{v} / \mathrm{v}$ ) against salt solution. Indeed, organic solvent directly contributes towards the degree by which the self-assembled membrane is plasticized, facilitating efflux of solvents from the polymersomes' lumen upon their exposure to hypertonic conditions. Eqn 1 describes the membrane's bending energy $\left(E_{\mathrm{b}}\right)$ in terms of its bending rigidity $k$.

$$
E_{\mathrm{b}}=\frac{k}{2} \oint\left(2 C-C_{0}\right)^{2} \mathrm{~d} A
$$

$C$ is the mean surface curvature and $C_{0}$ is spontaneous surface curvature. Although $k$ is valuable to the shape transformation process, there are few studies that actually demonstrate its importance. $K$ is directly affected by the intrinsic properties of the membrane, which is impacted by the amount of the organic solvent available during the shape transformation process. ${ }^{28}$ Dioxane plays an important role in plasticizing the membrane and thus, decreases bending rigidity $k$. According to eqn (1), decreasing $k$ would result in a lower $E_{\mathrm{b}}$ and thus, a faster shape transformation process. Moreover, when the membrane is highly flexible, out-flow of solvents from the polymersome lumen occurs at a faster rate - meaning increased volume reduction (at faster rate) and, ergo, longer tubes.

In order to confirm our hypothesis on the effect of both THF and dioxane on PDLLA-based polymersomes, we set out to study their extrusion (i.e. mechanical resizing), and the consecutive shape transformation of the resulted structures in presence of different THF : dioxane ratios. In general, extrusion of polymeric particles require high degree of membrane flexibility. Such extrusion process results in the formation of small polymersomes $(c a .100 \mathrm{~nm})$, which possess higher surface curvature when compared to their counterparts ( $c a .400 \mathrm{~nm}$ ) (Fig. S7 $\dagger$ ). Such size control over PEG-PDLLA polymersomes was previously realized by repeated extrusion of $c a .400 \mathrm{~nm}$ polymersomes, which are suspended in a higher content of organic solvent to water $(66 / 33 \mathrm{v} / \mathrm{v} \%)$. This solvent condition ensured membrane flexibility as well as permeability to allow efflux of solvent from the polymersomes lumen during their shape transformation into tubular morphologies. Without dioxane present in the solution, $\pm 400 \mathrm{~nm}$ polymersomes were successfully extruded into $\pm 150 \mathrm{~nm}$ polymersomes (Fig. 4A and S8, 9†); however, no shape transformation could be observed when osmotic shock was applied. These results highlight the role dioxane plays in reducing $k$ and $E_{\mathrm{b}}$, so that the shape transformation of high surface curvature structures can take place.

Higher aspect ratio particles could be obtained by further increasing the concentration of dioxane to a final THF : dioxane ratio of $30: 70$ (Fig. 4 and S8-10†), elongating spherical nanoparticles into prolates and eventually tubes demonstrating the role dioxane plays in plasticizing PDLLA membrane. At a mixture of THF and dioxane with a ratio $1: 4$, the shape transformation of the extruded polymersomes, in
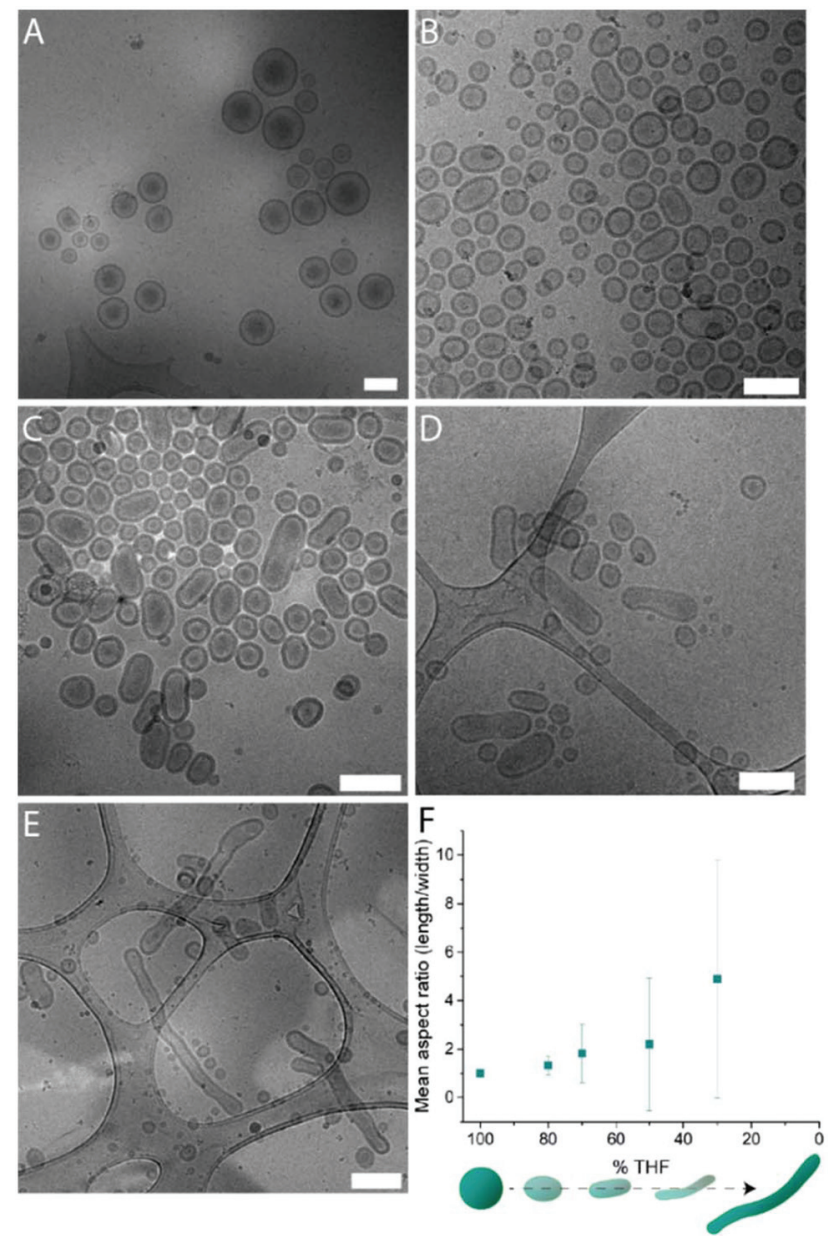

Fig. 4 Cryo-TEM images depicting the effect of increasing the concentration of dioxane on the shape transformation of extruded polymersomes (ca. $150 \mathrm{~nm}$ ) Respective THF: dioxane ratios (v/v\%) are: (A) $100: 0$, (B) $80: 20$, (C) $70: 30$, (D) $50: 50$ and (E) $30: 70$. The mean aspect ratio calculated from image analysis (F) depicts the increase in length-to-width variation with increasing dioxane ratios. Scale bars are $200 \mathrm{~nm}$. 
Table 2 Results from varying THF : dioxane ratios in extrusion of polymersomes and subsequent shape transformation

Solvent composition scheme

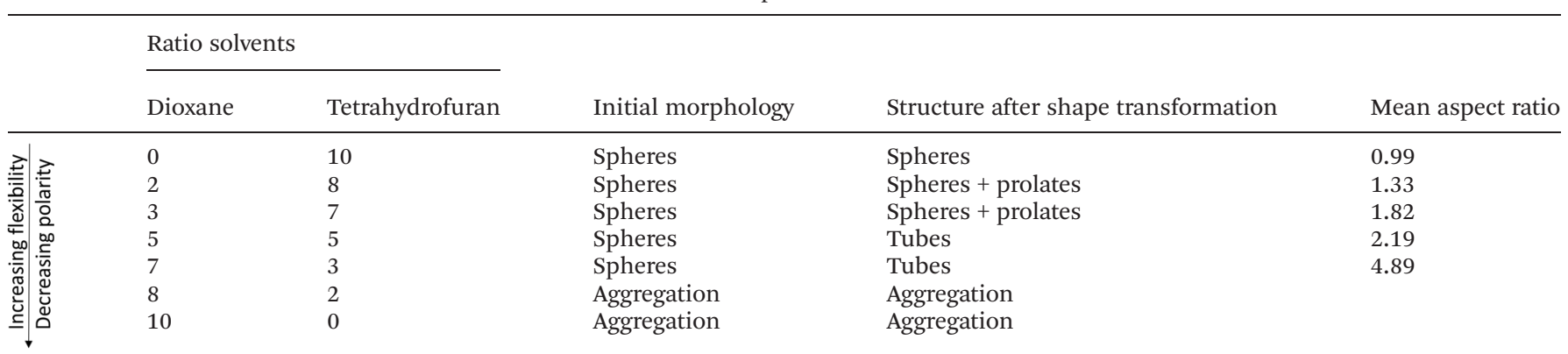

most cases, yielded a mixture of polymersomes and aggregates - again, highlighting the role of THF in modulating the polymer-solvent interactions and yielding a well-ordered membrane. Table 2 displays the results of the extrusion of polymersomes and their shape transformation with varying solvent composition.

From these experiments it is clear that the properties of the solvent are of utmost importance in modulating not only membrane formation but also the bending rigidity, either allowing or impeding shape transformation. Furthermore, it is clear that increasing solvent polarity (by increasing the amount of THF) is a significant driving factor in tailoring repulsive interactions between PEG-PDLLA polymer chains to yield stable assemblies. On the other hand, membrane flexibility is necessary to induce shape transformation; as evident by the formation of longer tubes at higher dioxane content.

\section{Conclusions}

The intricate interplay between dioxane and THF demonstrates that polymer swelling in cosolvents contributes immensely to the supramolecular structure of the self-assembled polymers and, in turn, morphology can be controlled. This provides us with means to control the morphology of PEG-PDLLA nanoparticles without directly adjusting the chemical composition of the copolymer, and therefore, facilitating easier techniques to control nanoparticle morphology for applications in bio(medical) chemistry.

\section{Conflicts of interest}

There are no conflicts to declare.

\section{Acknowledgements}

The authors would like to acknowledge the support from the NWO-NSFC Advanced Materials (project 792.001.015) and the National Natural Science Foundation of China (NSFC 51561135010).

\section{References}

1 L. Zhang and A. Eisenberg, Science, 1995, 268, 17281731.

2 A. C. Anselmo, C. L. Modery-Pawlowski, S. Menegatti, S. Kumar, D. R. Vogus, L. L. Tian, M. Chen, T. M. Squires, A. Sen Gupta and S. Mitragotri, ACS Nano, 2014, 8, 1124311253.

3 J. Wang, J. D. Byrne, M. E. Napier and J. M. Desimone, Small, 2011, 7, 1919-1931.

4 S. E. A. Gratton, P. A. Ropp, P. D. Pohlhaus, J. C. Luft, V. J. Madden, M. E. Napier and J. M. DeSimone, Proc. Natl. Acad. Sci. U. S. A., 2008, 105, 11613-11618.

5 P. Kolhar, A. C. Anselmo, V. Gupta, K. Pant, B. Prabhakarpandian, E. Ruoslahti and S. Mitragotri, Proc. Natl. Acad. Sci. U. S. A., 2013, 110, 10753-10758.

6 R. Salva, J. F. Le Meins, O. Sandre, A. Bruîlet, M. Schmutz, P. Guenoun and S. Lecommandoux, ACS Nano, 2013, 7, 9298-9311.

7 M. C. M. van Oers, W. S. Veldmate, J. C. M. van Hest and F. P. J. T. Rutjes, Polym. Chem., 2015, 6, 5358-5361.

8 R. Deng, M. J. Derry, C. J. Mable, Y. Ning and S. P. Armes, J. Am. Chem. Soc., 2017, 139, 7616-7623.

9 J. F. Le Meins, O. Sandre and S. Lecommandoux, Eur. Phys. J. E, 2011, 34, 1-17.

10 P. Schuetz, M. J. Greenall, J. Bent, S. Furzeland, D. Atkins, M. F. Butler, T. C. B. McLeish and D. M. A. Buzza, Soft Matter, 2011, 7, 749-759.

11 O. Terreau, L. Luo and A. Eisenberg, Langmuir, 2003, 19, 5601-5607.

12 A. Blanazs, J. Madsen, G. Battaglia, A. J. Ryan and S. P. Armes, J. Am. Chem. Soc., 2011, 133, 1658116587.

13 I. A. B. Pijpers, L. K. E. A. Abdelmohsen, Y. Xia, S. Cao, D. S. Williams, F. Meng, J. C. M. van Hest and Z. Zhong, Adv. Ther., 2018, 1800068.

14 Z. Li, J. Ma, N. S. Lee and K. L. Wooley, J. Am. Chem. Soc., 2011, 133, 1228-1231.

15 B. M. Discher, Y. Y. Won, D. S. Ege, J. C. M. Lee, F. S. Bates, D. E. Discher and D. A. Hammer, Science, 1999, 284, 11431146. 
16 Y. Zhu, B. Yang, S. Chen and J. Du, Prog. Polym. Sci., 2017, 64, 1-22.

17 J. D. Robertson, G. Yealland, M. Avila-Olias, L. Chierico, O. Bandmann, S. A. Renshaw and G. Battaglia, ACS Nano, 2014, 8, 4650-4661.

18 U. Borchert, U. Lipprandt, M. Bilang, A. Kimpfler, A. Rank, R. Peschka-Süss, R. Schubert, P. Lindner and S. Förster, Langmuir, 2006, 22, 5843-5847.

19 L. K. E. A. Abdelmohsen, D. S. Williams, J. Pille, S. G. Ozel, R. S. M. Rikken, D. A. Wilson and J. C. M. Van Hest, J. Am. Chem. Soc., 2016, 138, 9353-9356.

20 R. Cheng, F. Meng, C. Deng, H. A. Klok and Z. Zhong, Biomaterials, 2013, 34, 3647-3657.

21 P. L. Soo and A. D. I. Eisenberg, J. Polym. Sci., Part B: Polym. Phys., 2004, 42, 923-938.

22 E. Antoniou and P. Alexandridis, Eur. Polym. J., 2010, 46, 324-335.

23 V. V. Vasilevskaya, P. G. Khalatur and A. R. Khokhlov, Macromolecules, 2003, 36, 10103-10111.

24 D. J. Pochan, K. L. Wooley, H. Cui, S. Zhong and Z. Chen, Science, 2007, 317, 647-650.
25 K. T. Kim, J. Zhu, S. A. Meeuwissen, J. J. L. M. Cornelissen, D. J. Pochan, R. J. M. Nolte and J. C. M. Van Hest, J. Am. Chem. Soc., 2010, 132, 12522-12524.

26 C. K. Wong, A. F. Mason, M. H. Stenzel and P. Thordarson, Nat. Commun., 2017, 8, 1-10.

27 R. P. M. Lafleur, X. Lou, G. M. Pavan, A. R. A. Palmans and E. W. Meijer, Chem. Sci., 2018, 9, 6199-6209.

28 R. S. M. Rikken, H. Engelkamp, R. J. M. Nolte, J. C. Maan, J. C. M. van Hest, D. A. Wilson and P. C. M. Christianen, Nat. Commun., 2016, 7, 12606.

29 I. A. B. Pijpers, L. K. E. A. Abdelmohsen, D. S. Williams and J. C. M. Van Hest, ACS Macro Lett., 2017, 6, 1217-1222.

30 A. C. Wauters, I. A. B. Pijpers, A. F. Mason, D. S. Williams, J. Tel, L. K. E. A. Abdelmohsen and J. C. M. Van Hest, Biomacromolecules, 2018, 20, 177-183.

31 A. Choucair, C. Lavigueur and A. Eisenberg, Langmuir, 2004, 20, 3894-3900.

32 R. W. N. Nugroho, T. Pettersson, K. Odelius, A. Höglund and A.-C. Albertsson, Langmuir, 2013, 29, 8873-8881.

33 Y. Yu, L. Zhang and A. Eisenberg, Macromolecules, 1998, 9297, 1144-1154. 